\title{
The application of a feasible exercise training program in the office setting
}

\begin{abstract}
Background: Previous research support the claim that people who work in offices and sit for a long time are particularly prone to musculoskeletal disorders.

Objective: The main objective of this paper is to introduce an exercise training program designed to decrease muscle stiffness and pain that can be performed in the office setting.

Methods: Forty healthy office workers (age: $28 \pm 5.3$ years old; body mass: $87.2 \pm 10.2 \mathrm{~kg}$; height: $1.79 \pm 0.15 \mathrm{~m}$ ) apart from suffering from any sub-clinical symptoms of muscle and joint stiffness, and who had at least two years of experience in office work were chosen and randomly assigned to either an experimental group $(n=20)$ or a control group $(n=20)$. The experimental group performed the exercise training program three times a week for 11 weeks. The Cornell Musculoskeletal Discomfort Questionnaire was used to measure the pain levels in the neck, shoulders, and lower back areas. The Borg CR-10 Scale was used to measure their perceived exertion when doing the exercises, and a goniometer was used to measure the changes in range of motion (ROM) of the neck, hips, knees, and shoulders.
\end{abstract}

Results: The overall results indicated that the exercise program could significantly $(p<0.05)$ reduce the neck, shoulders, and lower back pains of the participants in the exercise group while those in the control group showed no improvement in those pains. There were significant $(p<0.05)$ increases in the ROM of the hips, the neck, both knees and shoulders in the exercise group. Participants showed significant $(p=0.011)$ decreases in perceived exertion scores after the exercises.

Conclusions: The exercise training program designed in this study not only can effectively reduce neck, shoulders, and lower back pains, but also can improve the ROM or flexibility of the office workers.

Keyword: Physical activity; Musculoskeletal disorders; Range of motion; Back pain 